\title{
Review of systems for recognising and responding to clinical deterioration in Singapore hospitals: a nationwide cross-sectional study
}

\author{
Sok Ying $\underline{\text { Liaw }}^{1}$, PhD, RN, Augustine $\underline{\text { Tee }}^{2}$, MBBS, FRCP, Guiller Augustin C $\underline{\text { Carpio }}^{3}$, DNM, MA, \\ Sophia Bee Leng Ang ${ }^{4}$, MBBS, MMed, Wei Ling $\underline{\text { Chua }}^{1}$, BSN(Hons), RN
}

\begin{abstract}
INTRODUCTION The Rapid Response System for recognising and responding to clinically deteriorating patients has been progressively implemented in acute care hospitals across the globe. This study sought to review the implementation of this system in acute public hospitals in Singapore.

METHODS A cross-sectional study using a face-to-face survey questionnaire was conducted.

RESULTS Five out of seven invited hospitals completed the questionnaire and rated the Rapid Response System as either high priority or essential, and indicated its importance over other patient safety indicators. Sensitivity and specificity of the triggering criteria and non-adherence to the escalation protocol were highlighted issues. Only two hospitals had a dedicated response team for providing emergency help to deteriorating ward patients. Limited manpower resources, unclear roles between the primary and response teams, and the potential deskilling of ward staff were reported barriers that inhibited the uptake of a response team. All hospitals had a committee that oversaw its system operation, provided training to ward staff, and used information technology to support the implementation.

CONCLUSION A variety of approaches have been taken to support the system of recognising and responding to clinical deterioration. This calls for a national approach to enable the standardisation of clinical processes, sharing of educational resources and multi-site evaluation.
\end{abstract}

Keywords: clinical deterioration, patient safety, physiological track and trigger systems, rapid response system, rapid response team

\section{INTRODUCTION}

Worldwide concerns over delays and failures of timely recognition and intervention in patients with early signs of clinical deterioration have driven the introduction of a hospital-wide patient safety initiative, known as the Rapid Response System (RRS), in acute hospitals. ${ }^{(1,2)}$ The aim of the RRS is to support frontline clinicians in recognising and responding promptly to clinical deterioration in general wards, with the ultimate goal of reducing unexpected cardiac arrests and admissions to the intensive care unit (ICU). ${ }^{(3)}$ The RRS bypasses the traditional hierarchical escalation of care by sanctioning bedside nurses and junior physicians to swiftly access medical assistance. ${ }^{(4)}$

Although existing evidence on the effectiveness of the RRS in achieving the stated aim is mixed, it is widely implemented in acute care hospitals across several countries, including Australia, the Netherlands, New Zealand, Scandinavia, the United Kingdom (UK), and the United States (US). ${ }^{(5-10)}$ An RRS generally has three key features: (1) an afferent limb to detect patient deterioration through a set of predetermined criteria known as physiological track and trigger systems (PTTSs) or early warning systems (EWSs); (2) an efferent limb consisting of a dedicated response team such as the Medical Emergency Team (MET) or Rapid Response Team (RRT); and (3) an administrative and quality improvement mechanism for process improvement and maintenance over time. ${ }^{(3,4)}$
There is a growing body of international literature describing nationwide implementation of the RRS. ${ }^{(7,8)}$ A nationwide review of the use of EWSs was conducted in all hospitals that admitted acute medical patients in the UK. Although all hospitals used an EWS at the point of entry to care, 11 different systems were used in London and five in Scotland. To address the disparity, a national EWS was recommended. (7) Similarly, a cross-sectional study in 20 public hospitals in New Zealand revealed that a large variance exists in the activation criteria to detect deteriorating adult patients, which called for the development of a national EWS and observational and response chart. ${ }^{(8)}$

Variations in the efferent limb of the RRS were also reported in nationwide studies conducted in the Netherlands, Australia and the US. In 63 of the 81 hospitals that responded to a nationwide survey in the Netherlands, only $78 \%$ had implemented an RRT, and $57 \%$ were physician-led and $89 \%$ were operated $24 / 7 .{ }^{(6)} \mathrm{A}$ survey of 39 (36.1\% response rate) Australian hospitals found that almost all RRTs were physician-led and operated 24/7. ${ }^{(5)}$ In contrast, a survey of RRTs in 31 hospitals (58\% response rate) in the US reported that the majority $(71 \%)$ used nurse-led teams and only $22 \%$ used a dedicated RRT nurse to respond to calls. Limited organisational resources and lack of funding were identified as factors that contributed to the variations in RRT composition. ${ }^{(11)}$ While anecdotal evidence indicates a progressive adoption

\footnotetext{
${ }^{1}$ Alice Lee Centre for Nursing Studies, Yong Loo Lin School of Medicine, National University of Singapore, ${ }^{2}$ Department of Respiratory and Critical Care Medicine, Changi General Hospital, ${ }^{3}$ Centre for Learning Environment and Assessment Development (CoLEAD), Singapore Institute of Technology, ${ }^{4}$ Department of Anaesthesia, National University Health System, Singapore

Correspondence: Dr Liaw Sok Ying, Associate Professor, Alice Lee Centre for Nursing Studies, Yong Loo Lin School of Medicine, National University of Singapore, Level 2, Clinical Research Centre, Block MD11, 10 Medical Drive, Singapore 117597.nurliaw@nus.edu.sg
} 
of the RRS by Singapore acute public hospitals, no published information is available. This study seeks to address this gap by exploring the existing recognition and response systems in Singapore acute public hospitals.

\section{METHODS}

A cross-sectional study was performed from September 2016 to January 2017 using a survey questionnaire after ethical approval was obtained from the institutional review boards (National Healthcare Group Domain Specific Review Board ref: 2016/00577). All six acute general public hospitals and a women's and children's hospital in Singapore were invited to participate in the study. These hospitals had bed capacities ranging from 590 to 1,785 beds. The authors only targeted public hospitals, as they were subjected to the guidance of broad government policy through the Ministry of Health. ${ }^{(12)}$

A survey questionnaire, adapted with permission from the Australian Commission on Safety and Quality in Health Care, ${ }^{(13)}$ was modified and used for this study. The questionnaire, which consists of both closed and open-ended questions, collects information about organisational systems for recognising clinical deterioration (afferent limb), systems for responding to clinical deterioration (efferent limb), and organisational systems for supporting the recognition of and response to deterioration (administrative and quality improvement limbs). It was validated by a panel of three local experts in the RRS, including two hospital clinicians and one university researcher. Based on the experts' reviews, modifications were made to some questions to allow for contextualisation to the local hospital practice. Two additional questions were added, which were related to the hospital's opinion of their priority on (a) addressing delays and failures to recognise and respond to deteriorating patients in the general ward, and (b) ranking seven patient safety indicators in order of importance, whereby ' 1 ' was the most important and ' 7 ' the least important.

An email invitation detailing the study, with the questionnaire and the consent form attached, was sent to each Chairman Medical Board of all seven hospitals. The hard copies were mailed via post immediately after the initial email invitation. In one month, a follow-up email was sent to remind the Chairman Medical Board of the opportunity to participate in the questionnaire. Hospitals were asked to contact the principal investigator to arrange for a one-time face-to-face meeting in the hospital's premises to complete the questionnaire, if they agreed to participate in the study. Administration of the questionnaire through a face-to-face approach allowed the clarification and elaboration of responses, particularly on open-ended comments. The face-to-face meetings were held with five teams of hospital personnel that championed their hospital's existing recognition and response systems. Only one questionnaire was completed for each hospital. Each face-to-face meeting lasted approximately 45 minutes to an hour, and was conducted by two investigators. The responses were recorded through field notes and sent back to the respondents for confirmation.

Descriptive statistics were used to analyse the quantitative data. Content analysis was performed on the responses to

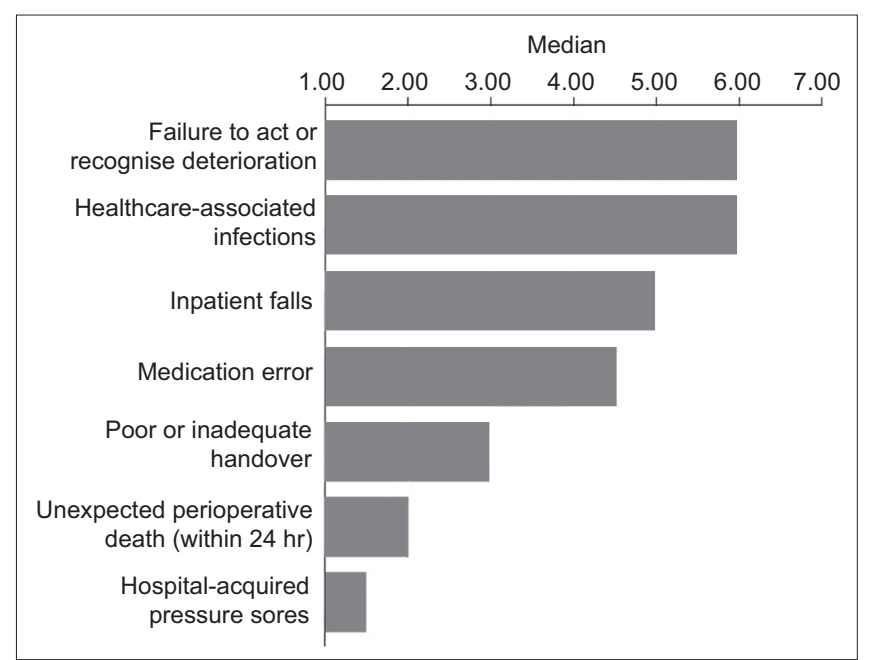

Fig. 1 Chart shows evaluation of the importance of patient safety indicators.

open-ended questions. Codes were generated from the responses and then merged to identify common themes. Two researchers independently analysed the data before coming together to compare their generated themes and reaching a consensus on the final set of themes. For the item on ranking seven patient safety indicators in order of importance, reversal scoring was used to calculate the median ranking score, with higher median value indicating high importance. One hospital declined ranking patient safety indicators and was thus excluded from the analysis.

\section{RESULTS}

Of the seven hospitals invited, 5 (71\%) participated in the study. One hospital declined to participate, as it did not have in place a recognition and response system for clinical deterioration, while the other hospital did not respond to the study invitation. All hospitals' champions that were present in each face-to-face meeting consisted of physicians, hospital patient safety leads and nurses. All physicians that were present were directly responsible for the RRS activities and three held instrumental leadership roles in their respective hospitals' patient safety or quality care.

All hospital respondents rated reducing delays and failures to recognise and respond to clinically deteriorating patients as either high priority (40\%) or essential (60\%). None responded with medium, low or not a priority. As shown in Fig. 1, hospitals ranked 'failure to act or recognise deterioration' as the most important patient safety indicator, which was on par with the indicator 'healthcare-associated infections', with a median rank score of 6 .

Table I shows the hospitals' written policies, protocols and guidelines on the measurement of vital signs observations for patients in general wards. These policies specify the minimum frequency and types of observation required. All five hospitals reported having formal escalation protocols that described actions to be taken when abnormal vital signs or other clinical deteriorations were observed in a patient. These escalation systems used a graded response, whereby different actions were required for different thresholds or levels of identified deterioration; typically, a senior doctor was informed at a higher threshold. The lack of adherence to the escalation protocol 
Table I. Systems for recognising and responding to clinical deterioration in Singapore hospitals $(n=5)$.

\begin{tabular}{|c|c|}
\hline Parameter & No. (\%) \\
\hline \multicolumn{2}{|l|}{ Afferent limb: systems to detect and trigger } \\
\hline Written policies on physiological observations & $5(100)$ \\
\hline Escalation protocol & $5(100)$ \\
\hline Track and trigger system & $5(100)$ \\
\hline Single/multiple parameter(s) & $2(40)$ \\
\hline Aggregate score & $1(20)$ \\
\hline Combined system & $2(40)$ \\
\hline Patient observation chart & $5(100)$ \\
\hline Colour-coded bands or zones & $1(20)$ \\
\hline Separate graphs for each triggering criterion & $1(20)$ \\
\hline Single graph of all trigger criteria & $1(20)$ \\
\hline Embedded alerts to prompt specific actions & $4(80)$ \\
\hline Electronic format & $5(100)$ \\
\hline Structured communication tool & $5(100)$ \\
\hline SBAR & $3(80)$ \\
\hline ISBAR & $2(40)$ \\
\hline SHARED & $0(0)$ \\
\hline \multicolumn{2}{|l|}{ Efferent limb: the response team } \\
\hline Formal Rapid Response System & $2(40)$ \\
\hline MET or RRT & $1(20)$ \\
\hline Incorporated within the 'code blue' team & $1(20)$ \\
\hline Consideration to implement & $2(40)$ \\
\hline \multicolumn{2}{|l|}{ Administrative and quality improvement mechanism } \\
\hline Staff with primary responsibility & $4(80)$ \\
\hline Committee to oversee the operation & $5(100)$ \\
\hline Regular training and education & $5(100)$ \\
\hline Collection of specific data & $5(100)$ \\
\hline No. of calls for emergency assistance to MET or other RRT & $3(60)$ \\
\hline No. of cardiac arrest & $4(80)$ \\
\hline No. of unplanned admissions to intensive care & $1(20)$ \\
\hline No. of unplanned readmissions to intensive care & $2(40)$ \\
\hline No. of deceased patients & $3(60)$ \\
\hline No. of deceased patients without a DNR or similar order & $1(20)$ \\
\hline No. of triggers and trigger scores & $2(40)$ \\
\hline
\end{tabular}

DNR: do not resuscitate; ISBAR: identity, situation, background, assessment recommendation; MET: medical emergency team; RRT: rapid response team SBAR: situation, background, assessment, recommendation; SHARED: situation, history, assessment, risk, expectation, documentation

by ward doctors and nurses were highlighted from the open comments of four hospitals (Table II).

Respondents from the five hospitals reported the use of a formal early warning, or a Track and Trigger System (TTS). These systems were implemented as early as 2009. Two hospitals reported using single- or multiple-parameter systems that were triggered when one or more specific criteria were met. One hospital employed an aggregate score system whereby a score was calculated from a number of specific criteria to determine the call for emergency assistance. Two hospitals used a combined system that not only included an aggregate score system but also a trigger that was response-based on any single parameter. All hospitals had a TTS built into their electronic patient observation chart. The majority (80\%) had embedded triggers and actions in their electronic patient observational charts.

As shown in Table II, the issue of sensitivity and specificity of the triggering criteria was raised by all hospital respondents. While two hospitals did not allow their ward staff to change the threshold of the triggering criteria, three hospitals permitted such a modification only by the medical staff. Four hospital respondents $(80 \%)$ emphasised the need to perform a holistic assessment to detect signs of deterioration that were beyond vital signs abnormalities.

Only two hospitals had a dedicated response team for providing emergency assistance, such as fluid resuscitation, medication administration, noninvasive ventilation, intubation and mechanical ventilation, to patients with deteriorating conditions. Of these two hospitals, one reported having a dedicated MET 24/7. The other hospital utilised a response team linked with a 'code blue' team to provide emergency assistance to peri-arrest patients and patients who developed a cardiac arrest. Both hospitals reported that their response teams, which constituted of ICU registrars, nurses and respiratory therapists, were based in the ICUs. Doctors were primarily responsible for leading the team. An after-hours, nurse-led service was reported by the hospital with a dedicated MET. Both hospitals reported that nurses and doctors in the ward, as well as other hospital staff (such as allied health professionals), could independently activate the response team. However, none reported that patients, family members and carers could call the response team. Only one hospital reported having formally-written guidelines that defined the roles and responsibilities of various stakeholders.

Of the three hospitals that reported not having a response team, two hospitals had plans to set up such teams. Three themes emerged from the respondents regarding the barriers and challenges that might inhibit the implementation of a response team (Table II): (1) manpower for the response team, particularly the need to provide continuous all-hours service; (2) role clarity between the primary and response teams; and (3) reliance on the response team, which can potentially lead to the deskilling of ward staff.

Four of the five hospitals reported that they had identified staff with the primary responsibility of developing, implementing, sustaining and monitoring their recognition and response system, and these staff typically had other concurrent clinical duties. Moreover, all hospitals reported that they had a committee that oversaw the operation of these systems. As highlighted by three hospital respondents, a major role of this committee was to get 'buy-in' from hospital staff to accept the system. In terms of staff training, all hospitals provided regular training to support doctors and nurses in the recognition of and response to clinical deterioration. However, three hospitals highlighted the challenge of orientating medical doctors due to their frequent rotations.

All five hospitals collected specific data to monitor the effectiveness of their recognition and response system. The most common data included the number of cardiac arrests (80\%) and deceased patients (60\%). Four hospitals reported the use of these data to track changes over time, identify possible improvement in 
Table II. Responses of participants to open-ended questions.

\begin{tabular}{|c|c|}
\hline Theme (\% of hospitals) & Examples of answers \\
\hline \multicolumn{2}{|c|}{$\begin{array}{l}\text { Afferent limb: systems to detect and trigger } \\
\text { Q: "Are there any comments about the systems in place for recognising clinical deterioration?" }\end{array}$} \\
\hline Sensitivity of trigger (100\%) & $\begin{array}{l}\text { "Hospital-wide standardisation of the trigger thresholds will compromise the sensitivity and } \\
\text { specificity of the triggers." (Hospital B) } \\
\text { "Triggering criteria used is generic and not specific for any disease." (Hospital E) }\end{array}$ \\
\hline Holistic assessment (80\%) & $\begin{array}{l}\text { "Looking at holistic patient assessment and patient signs." (Hospital C) } \\
\text { "Worried that clinicians will tend to overlook other signs and symptoms, e.g. cool and sweaty, chest } \\
\text { pain, that are beyond the vital signs." (Hospital E) }\end{array}$ \\
\hline Adherence to trigger protocol (80\%) & $\begin{array}{l}\text { "Junior medical and nursing staff's fear of hierarchy when activating response." (Hospital A) } \\
\text { "Reluctance of doctors to adhere to the trigger protocol and perform follow-up review of triggered } \\
\text { patients." (Hospital B) }\end{array}$ \\
\hline \multicolumn{2}{|c|}{$\begin{array}{l}\text { Efferent limb: the response team } \\
\text { Q: "Are there any comments about the systems in place for responding to clinical deterioration?" }\end{array}$} \\
\hline Manpower for response team ( $80 \%)$ & $\begin{array}{l}\text { "Main anticipated challenge is manpower and the ability to maintain a 24/7 service." (Hospital B) } \\
\text { "Lack of resources (manpower) for the dedicated response team." (Hospital D) }\end{array}$ \\
\hline $\begin{array}{l}\text { Role clarity between primary and } \\
\text { response teams }(40 \%)\end{array}$ & $\begin{array}{l}\text { "The need to have clear and defined roles and responsibilities in the primary team and the MET } \\
\text { during MET activation." (Hospital E) }\end{array}$ \\
\hline Reliance on response team (60\%) & $\begin{array}{l}\text { "...could potentially lead to ward staff being dependent on the MET." (Hospital B) } \\
\text { "The primary team may have the culture of leaving the MET to handle the deterioration." (Hospital E) }\end{array}$ \\
\hline \multicolumn{2}{|c|}{$\begin{array}{l}\text { Administrative and quality improvement mechanism } \\
\text { Q: "Are there any comments about the organisational system to support the recognition and response to clinical deterioration?" }\end{array}$} \\
\hline Acceptance to change (100\%) & $\begin{array}{l}\text { "The use of such systems needs the support of senior leadership and a working committee... } \\
\text { Cultural mindset change is required over time to fully utilise the system." (Hospital A) } \\
\text { "Getting buy-in from doctors." (Hospital B) }\end{array}$ \\
\hline Training (60\%) & $\begin{array}{l}\text { "Continuous training is needed to strengthen the afferent arm, especially since doctors are always } \\
\text { rotating on a three-monthly or monthly basis." (Hospital B) } \\
\text { "The challenge of training doctors due to frequent rotation. We need the nurses to be the anchor of } \\
\text { the trigger when doctors are always on rotation." (Hospital C) }\end{array}$ \\
\hline Information technology (IT) (80\%) & $\begin{array}{l}\text { "A tremendous amount of planning work needs to be done prior to the implementation, such as } \\
\text { building an IT system that does not add to the workload of nurses and doctors." (Hospital B) } \\
\text { "The use of IT enablers and infrastructure allows us to monitor all of the triggering criteria." (Hospital C) }\end{array}$ \\
\hline
\end{tabular}

MET: medical emergency team

services, and provide feedback to the ward and emergency teams. Four hospitals reported the use of information technology (IT) to facilitate the documentation and recognition of abnormal vital signs. Of these, two hospitals highlighted the use of IT to reduce unnecessary workload. Two hospitals also reported the use of IT to provide clinical decision support based on the hospital's escalation protocol.

\section{DISCUSSION}

Addressing the needs of deteriorating ward patients was clearly demonstrated to be of high priority for the Singapore acute hospitals that participated in this study. All five Singapore hospitals utilised TTSs to identify patients at risk of deterioration. The findings revealed that these hospitals were using different types of TTSs, adopting systems from either Australia or the UK. While the single-parameter system was developed from the MET model in Australia, ${ }^{(14)}$ the aggregate scoring system was associated with the EWS model that was originally developed in the UK. ${ }^{(15)}$ The variety of TTSs that exists to detect deteriorating patients within Singapore hospitals seems to reflect a lack of consensus or evidence on the 'best' system to fit the local hospital context. This variation has been highlighted in nationwide studies, including in Australia, Finland, New Zealand and the UK, which calls for a national standardisation of the TTS. $(5,7,8,10)$

Despite the individual uses of the TTS to suit hospital needs, the issue of sensitivity and specificity of the triggering criteria was raised in the study. While low sensitivity may imply that patients requiring immediate intervention are likely to be missed, ${ }^{(16)}$ high sensitivity may inadvertently desensitise the ward staff due to a high number of 'false-positive' patients. ${ }^{(17)}$ Several studies asserted the insufficiency of the TTS in picking up earlier signs of deterioration and, thus, should not be seen as replacing competent clinical judgement. ${ }^{(18,19)}$ For this reason, the importance of performing holistic assessment to detect more signs of deterioration was highlighted by the respondents in our study.

Surprisingly, two hospitals in the study reported that they did not allow ward staff to change the threshold of the triggering criteria. In contrast, several studies have highlighted the critical role of senior doctors in modifying the threshold of the triggering criteria for individual patients whose baseline values deviated from the norms. ${ }^{(17,20)}$ Furthermore, this inability to tailor threshold limits to accommodate individual medical conditions has been 
shown to cause ward nurses and doctors to be non-adherent to the thresholds of the activation criteria. ${ }^{(17)}$

A lack of adherence to the escalation protocol by ward staff was a concern raised by almost all hospital respondents. This can be attributed to a lack of understanding of physiological deterioration and unfamiliarity with the triggering criteria, ${ }^{(21,22)}$ highlighting the importance of staff education. ${ }^{(23)}$ Although all hospitals reported the implementation of RRS training for all ward doctors and nurses, no information was gathered on how the educational content was delivered. A previous study revealed a lack of effectiveness in conventional educational methods such as lecture, orientation sections and displaying of posters on the RRS. ${ }^{(23)}$ In addition to staff training, existing IT infrastructure, such as the electronic patient records, have been used by some hospitals to provide clinical decision support based on the hospital's escalation protocol. An important reason that hinders junior staff from following the escalation protocol could be the presence of traditional social and cultural barriers in the clinical areas. ${ }^{(24,25)}$ Further studies are suggested to aid in understanding individual cultural issues. ${ }^{(25)}$

As with TTSs, there was diversity in the hospitals' response to clinical deterioration. Over half of the hospitals in Singapore were without a response team, and this trend appears to contrast with the rapid growth of formal RRSs in Australia, ${ }^{(1)}$ the UK, ${ }^{(7)}$ and the US. The minority of Singapore hospitals that operate a formal RRS seems to adapt established international models to fit each local hospital with minimal national integration. According to a study in the US on the sustainability of RRTs, the flexibility in adopting an RRT model that fits the availability of resources and the needs of the organisation is critical for the success of RRT implementation. ${ }^{(11)}$ Furthermore, our findings indicate that more hospitals have considered creating a response team but are facing perceived barriers, including limited manpower resources, unclear role between the primary and response teams, and the potential deskilling of ward staff. Several studies conducted to examine these barriers have shown controversial findings. ${ }^{(18,26,27}$ More studies are needed to increase our understanding of ward staff's perceived barriers in order to identify strategies to overcome them. ${ }^{(28)}$

The importance of evaluating the RRS to improve patient outcomes has been well-recognised. Despite the range of clinical data collected by each hospital in this study, no data related to RRS in Singapore hospitals has been published in peer-reviewed journals. There should be an agreed core data set collected at a national level to allow comparison across hospitals and to provide guidance for the hospitals to improve their systems. ${ }^{(29)}$

This study's main strength was the administration of the questionnaire using face-to-face interviews with patient safety leads, allowing the interviewers to gather deeper information. However, the findings could be limited by the type of questions used in the questionnaire. The key limitation of this study was its inability to yield a $100 \%$ response rate, which may not provide a complete view of the national practice. Furthermore, the data was collected at a single time point; hence, the findings may not reflect current updates.
In conclusion, our study reviewed the use of RRSs in Singapore acute public hospitals. Recognising the high priority that all respondent hospitals have placed on supporting a system of care to identify and respond to deteriorating patients, this study raises questions on whether a national guideline for designing and implementing an RRS is required. The variations that exist in the RRSs of Singapore acute public hospitals may further call for a national-level response, much like the Australian Commission on Safety and Quality in Health Care and the UK's National Institute for Health and Clinical Excellence. A national approach to the design, implementation and evaluation of an RRS in Singapore will enable the standardisation of clinical processes, sharing of educational resources and multisite evaluation. This unifying strategy can potentially provide operational benefits and promote the development of research in this patient safety initiative in Singapore hospitals.

\section{ACKNOWLEDGEMENTS}

We thank the National University Health System Medical Publications Support Unit, Singapore, for providing editing services for this manuscript. This study was funded by the Centre for Health Services and Policy Research, National University Health System, Singapore.

\section{REFERENCES}

1. Australian Commission on Safety and Quality in Health Care. National Consensus Statement: Essential Elements for Recognising and Responding to Clinical Deterioration. Sydney, Australia: Australian Commission on Safety and Quality, 2010. Available at: https://www.safetyandquality.gov.au/wp-content/ uploads/2012/01/national_consensus_statement.pdf. Accessed June 18, 2016.

2. National Institute for Health and Clinical Excellence. Acutely ill patients in hospital: recognition of and response to acute illness in adults in hospital. July 2007. Available at: https://www.nice.org.uk/guidance/cg50/resources/cg50acutely-ill-patients-in-hospital-full-guideline3. Accessed June 18, 2016

3. Jones DA, DeVita MA, Bellomo R. Rapid-response teams. N Engl J Med 2011; 365:139-46.

4. Rao AD, DeVita MA. RRS's General Principles. In: DeVita MA, Hillman K, eds. Textbook of Rapid Response Systems: Concept and Implementation. 2nd ed. Cham, Switzerland: Springer International Publishing, 2017: 25-30.

5. ANZICS-CORE MET dose Investigators Jones D, Drennan K, et al. Rapid Response Team composition, resourcing and calling criteria in Australia. Resuscitation 2012; 83:563-7.

6. Ludikhuize J, Hamming A, de Jonge E, Fikkers BG. Rapid response systems in the Netherlands. Jt Comm J Qual Patient Saf 2011; 37:138-44, 97

7. Patterson C, Maclean F, Bell C, et al. Early warning systems in the UK: variation in content and implementation strategy has implications for a NHS early warning system. Clin Med (Lond) 2011; 11:424-7.

8. Psirides A, Hill J, Hurford S. A review of rapid response team activation parameters in New Zealand hospitals. Resuscitation 2013; 84:1040-4.

9. Stolldorf DP, Jones CB. Deployment of rapid response teams by 31 hospitals in a statewide collaborative. Jt Comm J Qual Patient Saf 2015; 41:186-91.

10. Tirkkonen J, Nurmi J, Olkkola T, Tenhunen J, Hoppu S. Cardiac arrest teams and medical emergency teams in Finland: a nationwide cross-sectional postal survey. Acta Aneaesthesiol Scand 2014; 58:420-7.

11. Stolldorf DP, Mion LC, Jones CB. A survey of hospitals that participated in a statewide collaborative to implement and sustain rapid response teams. J Healthc Qual 2016; 38:202-12.

12. Ministry of Health, Singapore. Hospital services. October 2015. Available at: https://www.moh.gov.sg/content/moh_web/home/our_healthcare_system/ Healthcare_Services/Hospitals.html. Accessed December 10, 2017.

13. Australian Commission on Safety and Quality in Health Care. Survey of recognition and response systems in Australia: summary of findings. September 2011. Available at: https://www.safetyandquality.gov.au/wp-content/ uploads/2012/01/survey_of_recognition_and_response_systems_-summary_ of_findings.pdf. Accessed November 15, 2015.

14. Hillman K, Parr M, Flabouris A, Bishop G, Stewart A. Redefining in-hospital resuscitation: the concept of the medical emergency team. Resuscitation 2001; 48:105-10 
15. Goldhill DR. The critically ill: following your MEWS. QJM 2001; 94:507-10.

16. Gao H, McDonnell A, Harrison DA, et al. Systematic review and evaluation of physiological track and trigger warning systems for identifying at-risk patients on the ward. Intensive Care Med 2007; 33:667-79.

17. Lydon S, Byrne D, Offiah G, Gleeson L, O'Connor P. A mixed-methods investigation of health professionals' perceptions of a physiological track and trigger system. BMJ Qual Saf 2016; 25:688-95.

18. Douglas C, Osborne S, Windsor C, et al. Nursing and medical perceptions of a hospital rapid response system: new process but same old game? J Nurs Care Qual 2016; 31:E1-E10.

19. Osborne S, Douglas C, Reid C, Jones L, Gardner G; RBWH Patient Assessment Research Council. The primacy of vital signs--acute care nurses' and midwives' use of physical assessment skills: a cross sectional study. Int J Nurs Stud 2015; 52:951-62.

20. Elliott D, Allen E, Perry L, et al. Clinical user experiences of observation and response charts: focus group findings of using a new format chart incorporating a track and trigger system. BMJ Qual Saf 2015; 24:65-75.

21. De Meester K, Van Bogaert PV, Clarke SP, Bossaert L. In-hospital mortality after serious adverse events on medical and surgical nursing units: a mixed methods study. J Clin Nurs 2012; 22:2308-17.

22. Ludikhuize J, Smorenburg SM, de Rooij SE, de Jonge E. Identification of deteriorating patients on general wards; measurement of vital parameters and potential effectiveness of the Modified Early Warning Score. J Crit Care 2012; 27:424.e7-13.

23. Sandroni C, Cavallaro F. Failure of the afferent limb: a persistent problem in rapid response systems. Resuscitation 2011; 82:797-8.

24. Jones D, Baldwin I, McIntyre T, et al. Nurses' attitudes to a medical emergency team service in a teaching hospital. Qual Saf Health Care 2006; 15:427-32.

25. Shearer B, Marshall S, Buist MD, et al. What stops hospital clinical staff from following protocols? An analysis of the incidence and factors behind the failure of bedside clinical staff to activate the rapid response system in a multi-campus Australian metropolitan healthcare service. BMJ Qual Saf 2012; 21:569-75.

26. Benin AL, Borgstrom CP, Jenq GY, Roumanis SA, Horwitz LI. Defining impact of a rapid response team: qualitative study with nurses, physicians and hospital administrators. BMJ Qual Saf 2012; 21:391-8.

27. Radeschi G, Urso F, Campagna S, et al. Factors affecting attitudes and barriers to a medical emergency team among nurses and medical doctors: a multi-centre survey. Resuscitation 2015; 88:92-8.

28. Chua WL, See MTA, Legio-Quigley $\mathrm{H}$, et al. Factors influencing the activation of the rapid response system for clinically deteriorating patients by frontline ward clinicians: a systematic review. Int J Qual Health Care 2017; 29:981-98.

29. Pedersen A, Psirides A, Coombs M. Models and activities of critical care outreach in New Zealand hospitals: results of a national census. Nurs Crit Care 2016; 21:233-42. 